\title{
RESTRICTIONS TO CONTINUOUS FUNCTIONS AND BOOLEAN ALGEBRAS
}

\author{
IRENEUSZ RECLAW
}

(Communicated by Andrew M. Bruckner)

\begin{abstract}
We show that every Borel function $f: \mathbb{R} \rightarrow \mathbb{R}$ is continuous on a set $A \notin \mathscr{J}$ if $B(\mathbb{R}) / \mathcal{J}$ is weakly distributive. We also show that CCC is not sufficient. We investigate some other conditions considering the problem of restrictions to continuous functions.
\end{abstract}

There are many theorems that say some kinds of functions have restrictions to continuous functions on large sets (see, e.g., [Bl, $\mathrm{Br}, \mathrm{BP}, \mathrm{SZ}]$ ). We recall a few.

Theorem A. For every Lebesgue measurable function $f: \mathbb{R} \rightarrow \mathbb{R}$ there is a set $D$ of positive measure such that $f \mid D$ is continuous.

Theorem B. For every function with the Baire property $f: \mathbb{R} \rightarrow \mathbb{R}$ there is a set $D$ of second category and with the Baire property such that $f \mid D$ is continuous.

Theorem C (Sierpinski and Zygmund [SZ]). There is a function $f: \mathbb{R} \rightarrow \mathbb{R}$ such that $f$ is not continuous on any set of size continuum.

Theorem D (Blumberg [Bl]). For every function $f: \mathbb{R} \rightarrow \mathbb{R}$ there is a countable dense set $D$ such that $f \mid D$ is continuous.

Let us try to express all these theorems in a common language.

Definition 1. Let $\mathscr{A}$ be a $\sigma$-algebra on a topological space $X$ and $\mathscr{J} \subseteq \mathscr{A}$ be a proper ideal. We say that $(\mathscr{A}, \mathscr{J})$ has CRP (continuous restrictions property) if for every $\mathscr{A}$-measurable function $f: X \rightarrow \mathbb{R}$ there exists $D \in \mathscr{A} \backslash \mathscr{J}$ such that $f \mid D$ is continuous.

Let $L$ be the $\sigma$-algebra of Lebesgue measurable sets; let $L_{0}$ be the $\sigma$-ideal of Lebesgue negligible sets; let $B_{w}$ be the $\sigma$-algebra of sets with Baire property; and let $F C$ be the $\sigma$-ideal of sets of first category.

As simple corollaries we get

Received by the editors September 29, 1991.

1991 Mathematics Subject Classification. Primary 26A15, 06E10; Secondary 28A10, 04A15.

Key words and phrases. Continuity, weak distributivity, Boolean algebra, Lusin set.

Most of the results in this paper were obtained when the author was visiting Auburn University, supported by the Kosciuszko Foundation. 
Corollary 1.1. Pairs $\left(L, L_{0}\right),\left(B_{w}, F C\right),\left(P(\mathbb{R}),[\mathbb{R}]^{<\omega}\right),\left((s),\left(s_{0}\right)\right)($ see $[\mathrm{Ma}])$ have CRP.

Corollary 1.2. The pair $\left(P(\mathbb{R}),[\mathbb{R}]^{<2^{\omega}}\right)$ does not have $C R P$.

Throughout this paper we say that a function $f: Z \rightarrow \mathbb{R}$ is $B$-measurable if it is measurable with respect to $B(Z)$, the $\sigma$-algebra generated by all closed $G_{\delta}$-sets.

We say that a subset $L \subseteq \mathbb{R}$ is a Lusin (Sierpiński) set if $L$ is uncountable and its intersection with every set of first category (of measure zero) is countable. A set is a $\lambda$-set if every countable set is relatively $G_{\delta}$. A set $X \subseteq \mathbb{R}^{n}$ is universally null if $\mu^{*}(X)=0$ for every continuous Borel measure $\mu$ on $\mathbb{R}^{n}$.

In this paper we will investigate the property CRP considering properties of the quotient Boolean algebra $\mathscr{A} / \mathscr{J}$. First we will consider $\mathscr{A}=B(\mathbb{R})$ and $\mathscr{J} \subseteq B(\mathbb{R})$ such that $B(\mathbb{R}) / \mathscr{J}$ is CCC. We see that for $\mathscr{J}=L_{0}$ and $\mathscr{J}=F C$, $(B(\mathbb{R}), \mathscr{J})$ has CRP.

Theorem 1. There is a $\sigma$-ideal $\mathscr{J} \subseteq B(\mathbb{R})$ such that $B(\mathbb{R}) / \mathscr{J}$ has $C C C$ and $(B(\mathbb{R}), \mathcal{J})$ does not have $C R P$. Moreover, $B(\mathbb{R}) / \mathscr{J}$ is isomorphic to $B(\mathbb{R}) / F C$.

A similar result was shown independently in [CMPS].

Proof. Lusin [L] showed that there is a continuous one-to-one function $g$ : $\omega^{\omega} \rightarrow \omega^{\omega}$ such that for every perfect set $D \subseteq \omega^{\omega}$ there is an open dense set $U \subseteq \omega^{\omega}$ such that $g[U] \cap D$ is first category in $D$.

Let $B=g\left[\omega^{\omega}\right]$ and $f=g^{-1} \mid B$ and let $\mathscr{J}=\{g[F]: F$ is first category . Since $g$ is a Borel isomorphism, we have that $f$ is Borel and $\mathcal{J}$ is a $\sigma$-ideal such that $B(B) / \mathcal{J}$ is CCC. Let us assume that there is a set $X \in B(B) \backslash \mathscr{J}$ such that $f \mid X$ is continuous and hence, a homeomorphism. We can assume that $X$ is dense in itself. Then $D=\bar{X}$ is perfect. Let $U \subseteq \omega^{\omega}$ be an open dense set such that $g[U] \cap D$ is first category in $D$. Thus $A=g[U] \cap X$ is first category in $X$. Since $f \mid X$ is a homeomorphism, $f[A]$ is first category in $f[X]$, and, so, in $\omega^{\omega}$. Thus $A \in \mathscr{J}$ because $A=g[f[A]] . X \backslash A$ also belongs to $\mathscr{J}$ because $X \backslash A=g\left[\left(\omega^{\omega} \backslash U\right)\right] \cap X$. So $X \in \mathscr{J}$-a contradiction.

Now we can extend a function $f$ to a Borel function $h$ defined on $\mathbb{R}$ and let $\mathscr{J}^{*}=\{C \in B(\mathbb{R}): C \cap B \in \mathcal{J}\}$. Then the function $h$ and the $\sigma$-ideal $\mathscr{J}^{*}$ have the required properties.

From this theorem we have that the properties of $B(\mathbb{R}) / F C$ are too weak to imply that $(B(\mathbb{R}), \mathscr{J})$ has CRP. So we will look more carefully at the measure Boolean algebra. Of course if $\mathscr{A}$ is the $\sigma$-algebra of measurable sets with respect to a finite measure $\mu$ and $\mathscr{J}$ is the $\sigma$-ideal of $\mu$-measure zero sets then $(\mathscr{A}, \mathscr{J})$ has CRP. We consider a weaker condition: weak distributivity.

Definition 2.1. $\mathscr{C} \subseteq \mathscr{A}$ is predense if for every $D \in \mathscr{A} \backslash \mathscr{J}$ there is $C \in \mathscr{C}$ such that $C \cap D \notin \mathscr{J}$.

Definition 2.2. $\mathscr{A} / \mathscr{J}$ is weakly distributive if for every $D \in \mathscr{A} \backslash \mathscr{J}$ and for every sequence of predense families $\mathscr{C}_{n} \subseteq \mathscr{A}$ there is sequence of finite subfamilies $\mathscr{C}_{n}^{\prime} \subseteq \mathscr{C}_{n}$ such that $D \cap \bigcap_{n} \cup \mathscr{C}_{n}^{\prime} \notin \mathscr{J}$.

Definition 3. Let $\mathscr{F} \subseteq \mathbb{R}^{X}$. Then let $\mathscr{F}_{c}$ be the smallest family of functions containing $\mathscr{F}$ closed under pointwise limits.

We write $f_{n} \rightrightarrows f$ if $f_{n}$ is converging uniformly to $f$. 
Theorem 2. Let $\mathscr{F}$ be a family of $\mathscr{A}$-measurable functions. If $\mathscr{A} / \mathscr{J}$ is weakly distributive then for every $D \in \mathscr{A} \backslash \mathscr{J}$ and for every $f \in \mathscr{F}_{c}$ there is a sequence of functions $\left\{f_{n}: n \in \omega\right\} \subseteq \mathscr{F}$ and there is $C \in \mathscr{A}$ such that $C \cap D \notin \mathscr{J}$ and $f_{n} \rightrightarrows f$ on $C \cap D$, or, equivalently, $\left\{C: \exists_{\left\{f_{n}: n \in \omega\right\} \subseteq \mathscr{F}} f_{n} \rightrightarrows f\right.$ on $\left.C\right\}$ is predense for every $f \in \mathscr{F}_{c}$.

Proof. We can define inductively $\mathscr{F}_{\alpha}$ as the family of all pointwise limits of functions from $\bigcup_{\beta<\alpha} \mathscr{F}_{\beta}$. It is easy to see that $\mathscr{F}_{c}=\bigcup_{\beta<\omega_{1}} \mathscr{F}_{\beta}$. We inductively show that for every function $f \in \mathscr{F}_{c}$ there is a family $\mathscr{C}_{f}$ such that $\mathscr{C}_{f}$ is closed under finite unions, predense, and for every $C \in \mathscr{C}_{f}$ there is a sequence $\left\{f_{n}\right.$ : $n \in \omega\} \subseteq \mathscr{F}$ converging uniformly to $f$ on $C$. Let a function $f \in \mathscr{F}_{\alpha}$. Then there is a sequence of functions $\left\{f_{n}: n \in \omega\right\} \subseteq \bigcup_{\beta<\alpha} \mathscr{F}_{\beta}$ such that $f_{n} \rightarrow f$. Let $\mathscr{E}_{k}=\left\{A_{n k}: n, k \in \omega\right\}$ where $A_{n k}=\left\{x: \forall_{j \geq n}\left|f(x)-f_{j}(x)\right|<1 / k\right\} . \mathscr{E}_{k}$ is predense. So since $A_{n k}$ is increasing with respect to $n$ and $\bigcup_{n} A_{n k}=X$, for $D \notin \mathcal{J}$ there is a subsequence $n_{k}$ such that $D \cap \bigcap_{k} A_{n_{k} k} \notin J$. Observe that $f_{n}$ converges uniformly to $f$ on $D \cap \bigcap_{k} A_{n_{k} k}$. So $\mathscr{E}=\left\{C: f_{n}\right.$ is converging uniformly to $f$ on $C$ \} is predense and closed under finite unions. Let $\mathscr{C}_{f_{n}}$ be predense families given by the induction hypothesis. For every $D \in \mathscr{A} \backslash \mathscr{J}$ there is a sequence $\mathscr{C}_{f_{n}}^{\prime} \subseteq \mathscr{C}_{f_{n}}$ of finite families such that $D \cap \bigcap_{n} \cup \mathscr{C}_{f_{n}}^{\prime} \notin \mathscr{J}$. The family of sets of the form $\bigcap_{n} \cup \mathscr{C}_{f_{n}}^{\prime}$ is predense and for every $n$ there is a sequence $\left\{f_{n k}: k \in \omega\right\} \subseteq \mathscr{F}$ such that $f_{n k}$ is uniformly converging to $f_{n}$ on $\bigcap_{n} \cup \mathscr{C}_{f_{n}}^{\prime}$. Let $\mathscr{C}$ be the closure under finite unions of that family; it also has that property. Let $\mathscr{C}_{f}$ be the closure under finite unions of $\{A \cap C: A \in \mathscr{E}$ and $C \in \mathscr{C}\}$. We can see that for every element $C$ of $\mathscr{C}_{f}$, for every $n$ there is a sequence $\left\{f_{n k}: k \in \omega\right\} \subseteq \mathscr{F}$ such that $f_{n k}$ is uniformly converging to $f_{n}$ on $C$ and $f_{n}$ is uniformly converging to $f$ on $C$. So there is a sequence $\left\{f_{n k_{n}}: n \in \omega\right\} \subseteq \mathscr{F}$ such that $f_{n k_{n}}$ is uniformly converging to $f$ on $C$.

Corollary 2. Let $X$ be a normal topological space and $\mathscr{J} \subseteq B(X)$ such that $B(X) / \mathscr{J}$ is weakly distributive. Then the pair $(B(X), \mathscr{J})$ has $C R P$.

Proof. Observe that every $B$-measurable function $f \in C(X)_{\mathrm{c}}$ where $C(X)$ is the family of all continuous functions on $X$. So the family of all sets on which the $B$-measurable function $f$ is a uniform limit of continuous functions is predense.

The next theorem shows that we can consider more general $\sigma$-algebras.

Lemma 1. Let $\mathscr{A}$ be a $\sigma$-algebra and $\mathscr{J}$ a $\sigma$-ideal such that $\mathscr{A} / \mathscr{J}$ is weakly distributive and for every $C \in \mathscr{A} \backslash \mathscr{J}$ there is $D \in B(X) \backslash \mathcal{J}$ such that $D \subseteq C$. Then for every $\mathscr{A}$-measurable function $f: X \rightarrow \mathbb{R}$ the set $\{D \in B(X): f \mid D$ is $B$-measurable $\}$ is predense.

Proof. Let $\left\{O_{n}: n \in \omega\right\}$ be a basis of $\mathbb{R}$. For every $\mathscr{A}$-measurable function we can define $\mathscr{C}_{n}=\left\{C \in B(X): C \subseteq f^{-1}\left[O_{n}\right]\right.$ or $\left.C \subseteq X \backslash f^{-1}\left[O_{n}\right]\right\}$. For every $n$, $\mathscr{C}_{n}$ is predense, so for every $D \in \mathscr{A} \backslash \mathscr{J}$ there is a sequence of finite subfamilies $\mathscr{C}_{n}^{\prime} \subseteq \mathscr{C}_{n}$ such that $D \cap \bigcap_{n} \cup \mathscr{C}_{n}^{\prime} \notin \mathscr{J}$. Observe that $f^{-1}\left[O_{n}\right] \cap \bigcap_{n} \cup \mathscr{C}_{n}^{\prime}=$ $\bigcap_{n} \cup \mathscr{C}_{n}^{\prime} \cap \bigcup \mathscr{C}_{n}^{\prime \prime}$ where $\mathscr{C}_{n}^{\prime \prime} \subseteq \mathscr{C}_{n}^{\prime}$. So $f \mid \bigcap_{n} \cup \mathscr{C}_{n}^{\prime}$ is $B$-measurable.

From the theorems above we get:

Theorem 3. Let $X$ be a normal topological space. Let $\mathscr{A}$ be a $\sigma$-algebra, $\mathscr{J}$ a $\sigma$-ideal such that $\mathscr{A} / \mathscr{J}$ is weakly distributive, and for every $C \in \mathscr{A} \backslash \mathscr{J}$ there is a $D \in B(X) \backslash \mathscr{J}$ such that $D \subseteq C$. Then the pair $(\mathscr{A}, \mathscr{J})$ has $C R P$. 
Definition 4. We say that the sequence $f_{n}: X \rightarrow \mathbb{R}$ is quasinormally converging to $f\left(f_{n} \stackrel{Q N}{\rightarrow} f\right)$ if $\exists_{\varepsilon_{n} \backslash 0} \forall_{x \in X} \exists_{k} \forall_{n \geq k}\left|f_{n}(x)-f(x)\right|<\varepsilon_{n}$.

It is known (see [BRR]) that $f_{n} \stackrel{Q N}{\rightarrow} f$ iff $X=\bigcup_{k} X_{k}$ and $f_{n} \rightrightarrows f$ on $X_{k}$ for each $k$.

We say that a property is satisfied a.e. (almost everywhere) if it is satisfied on $X \backslash D$ for a set $D \in \mathcal{J}$.

Lemma 2. Let $X$ be a normal topological space and $\mathscr{J} \subseteq B(X)$ such that $B(X) / \mathcal{J}$ is weakly distributive and $C C C$. Then

(a) If $f_{n} \rightarrow f$ then $f_{n} \stackrel{Q N}{\rightarrow} f$ a.e.

(b) If $f_{n} \rightarrow f$ and $f_{n k} \rightarrow f_{n}$ then there is a subsequence $k_{n}$ such that $f_{n k_{n}} \stackrel{Q N}{\rightarrow} f$ a.e.

$\operatorname{Proof}$ (due to [BRR] or [W]). (a) Let $A_{n k}=\left\{x: \forall_{j \geq n}\left|f(x)-f_{j}(x)\right|<1 / k\right\}$ and $\mathscr{A}_{n}=\left\{A_{n k}: k \in \omega\right\}$. Then the $\mathscr{A}_{n}$ are predense, so as in the proof of Theorem 2 there is predense $\mathscr{C}$ such that for every $C \in \mathscr{C}, f_{n} \rightrightarrows f$ on $C$. By CCC there is a countable $\mathscr{C}^{\prime} \subseteq \mathscr{C}$ such that for every $C \in \mathscr{C}^{\prime}, f_{n} \rightrightarrows f$ on $C$ and $X \backslash \cup \mathscr{C}^{\prime} \in \mathcal{J}$.

(b) From (a) we get that there are countable $\mathscr{A}_{n}$ such that $f_{n k} \rightrightarrows f_{n}$ on $C$ for every $C \in \mathscr{A}_{n}$, and a countable $\mathscr{B}$ for every $C \in \mathscr{B}, f_{n} \rightrightarrows f$ on $C$, and $\left(X \backslash\left(\cap_{n} \cup \mathscr{A}_{n} \cap \bigcup \mathscr{B}\right)\right) \in \mathscr{J}$. So there is a countable $\mathscr{C}$ such that for every $C \in \mathscr{C}, f_{n} \rightrightarrows f$ on $C, f_{n k} \rightrightarrows f_{n}$ on $C$, and $X \backslash \cup \mathscr{C} \in \mathcal{J}$. We can choose by induction $k_{n}$ such that for every $C \in \mathscr{C}, f_{n k_{n}} \rightrightarrows f$ on $C$.

Corollary 3. Let $X$ be a normal topological space. Let $\mathscr{A}$ be a $\sigma$-algebra, $\mathcal{J}$ a $\sigma$-ideal such that $\mathscr{A} / \mathscr{J}$ is weakly distributive and CCC, and for every $C \in \mathscr{A} \backslash \mathscr{J}$ there is a $D \in B(X) \backslash \mathcal{J}$ such that $D \subseteq C$. Then for every $\mathscr{A}$ measurable function $f: X \rightarrow \mathbb{R}$ there is a $B \in \mathcal{J}$ and a sequence $f_{n}: X \rightarrow \mathbb{R}$ of continuous functions such that $f_{n}: X \backslash B \stackrel{Q N}{\rightarrow} \mathbb{R}$.

Proof. First by Lemma 1 we get that there is a set $F \in \mathscr{J}$ such that $f \mid X \backslash F$ is $B$-measurable because a countable union of Borel functions on Borel sets is a Borel function. As in the proof of Theorem 2 we show by induction with respect to the Baire class of the Borel function $f: X \rightarrow \mathbb{R}$ that there is a sequence $f_{n}: X \rightarrow \mathbb{R}$ of continuous functions and $B \in \mathcal{J}$ such that $f_{n} \mid X \backslash B \stackrel{Q N}{\rightarrow} \mathbb{R} . f$ is a pointwise limit of functions $f_{n}$ of lower Baire class. By induction we have that $f_{n}$ is a pointwise limit of continuous functions a.e. and by Lemma 2 we get that $f$ is also a quasi-normal limit of continuous functions a.e.

Now we will consider an ideal of the form $\mathscr{J}=[X]^{<\kappa}$ where $\kappa$ is a cardinal. Let $X \subseteq \mathbb{R}$ such that $|X|=\kappa$.

Fact 1. (a) The pair $\left(P(X),[X]^{<\kappa}\right)$ does not have CRP if $\kappa=2^{\omega}$ (Sierpinski and Zygmund [SZ]).

(b) The pair $\left(P(X),[X]^{<\kappa}\right)$ has CRP if $\omega<\kappa<2^{\omega}$ under MA (Shinoda [Sh]).

The following theorem gives us independence of the result (b).

Theorem 4 (Gruenhage). Let $V$ satisfy $2^{\omega}=\kappa$ and let $\lambda \geq \kappa$ be a cardinal with $\lambda^{\omega}=\lambda$. If $\lambda$ Cohen or random reals are added to $V$ then in the extension 
$\exists F: \mathbb{R} \rightarrow \mathbb{R}$ such that $F \mid X$ is not Borel whenever $X$ is an uncountable subset of $\mathbb{R}$.

Proof. It suffices to prove the proposition with $2^{\omega}$ in place of $\mathbb{R}$. We will verify the Cohen reals case; the random reals case is analogous, using the measure algebra of $2^{\lambda \times \omega}$ in place of $\operatorname{Fn}(\lambda \times \omega, 2)$.

Let $P=\operatorname{Fn}(\lambda \times \omega, 2)$, and let $G$ be a generic filter. Then $V[G]$ satisfies $2^{\omega}=\lambda$. For each $x \in 2^{\omega} \cap V[G]$, there is a countable set $C_{x} \subseteq \lambda$ such that $x \in V\left[G \cap \operatorname{Fn}\left(C_{x} \times \omega, 2\right)\right]$. One can define a one-to-one function $\Theta: \omega^{\omega} \rightarrow$ $\lambda$ such that $\forall x \boldsymbol{\Theta}(x) \notin C_{x}$. Let $f_{\boldsymbol{\Theta}(x)}=\bigcup G(\boldsymbol{\Theta}(x), \cdot): \omega \rightarrow 2$, and let $F(x)=f_{\Theta(x)}$. Suppose $F \mid X$ is Borel and $X$ is uncountable. $F \mid X$ extends to some $F^{\prime}: Y \rightarrow 2^{\omega}$, where $Y$ is Borel. Then there exists a countable $E \subseteq \lambda$ such that $F^{\prime} \in V[G \cap \mathrm{Fn}(E \times \omega, 2)]$. Choose $x \in X$ such that $\Theta(x) \notin E$. Then $F^{\prime}, x \in V\left[G \cap \operatorname{Fn}\left(\left(E \cup C_{x}\right) \times \omega, 2\right)\right]$, but $F^{\prime}(x)=F(x)=f_{\Theta(x)} \notin$ $V\left[G \cap \mathrm{Fn}\left(\left(E \cup C_{x}\right) \times \omega, 2\right)\right]$ since $\Theta(x) \notin E \cup C_{x}$. This is a contradiction.

Of course this theorem implies that the pair $\left(P(X),[X]^{\leq 2 \omega} 1\right)$ does not have CRP for every $X \subseteq \mathbb{R}$ of size $\omega_{1}$. It is also worthwhile to see the following facts.

Fact 2. If $X \subseteq \mathbb{R}$ is a Sierpinski set or a Lusin set of size $\omega_{1}$ then there is a set $Y \subseteq \mathbb{R}$ such that for every one-to-one function $f: X \rightarrow Y, f$ is not continuous on any uncountable subset of $X$.

Proof. Let $Y \subset \mathbb{R}$ be a universally null set of size $\omega_{1}$. Then let $f$ be a one-toone function $f: X \rightarrow Y$ and suppose that $f$ is continuous on an uncountable subset of $X$. Then this subset in universally null; but a Sierpiński set does not contain an uncountable subset of measure zero.

In the case of a Lusin set $Y$ is an uncountable $\lambda$-set.

Fact 3. If $L \subseteq \mathbb{R}^{2}$ is a Lusin set or Sierpinski set in $\mathbb{R}^{2}$ then for every function $f$ : $p_{1}(L) \rightarrow p_{2}(L)$ such that $\operatorname{graph}(f) \subseteq L, f$ is not continuous on any uncountable subset of $p_{1}(L)$.

Proof. The graph of any continuous function is of first category and measure zero in $\mathbb{R}^{2}$, so its intersection with $L$ is countable.

For the case of the $\sigma$-algebra $B(X)$ we recall the following results.

Theorem 5 (Sierpinski [S]). If there is a Lusin set $L \subseteq \mathbb{R}$ then there is a set $X$ of size $|L|$ such that $\left(B(X),[X]^{<\omega_{1}}\right)$ does not have CRP.

Proof. As in the proof of Theorem 1 we take the Luzin function $g: \omega^{\omega} \rightarrow \omega^{\omega}$. Let us take a Lusin set $L \subseteq \omega^{\omega}$ and let $X$ be $g[L]$. Then $\mathscr{J} \cap X=[X] \leq \omega$ for the ideal $\mathscr{J}$ from Theorem 1 . So $g^{-1} \mid X$ is not continuous on any uncountable set.

Theorem 6 (Cichon and Morayne [CM]). It is consistent that, for every set $X \subseteq \mathbb{R}$ of size $2^{\omega},\left(B(X),[X]^{<2^{\omega}}\right)$ has $C R P$.

\section{ACKNOWLEDGMENT}

The author would like to express his thanks to J. B. Brown, R. Beaudoin, G. Gruenhage, and J. Jasiński for fruitful discussions. 


\section{REFERENCES}

[Bl] H. Blumberg, New properties of all real functions, Trans. Amer. Math. Soc. 24 (1922), 113128.

[Br] Jack B. Brown, Variations on Blumberg's Theorem, Real Anal. Exchange 9 (1983), 123-137.

[BP] Jack B. Brown and K. Prikry, Variations on Lusin's theorem, Trans. Amer. Math. Soc. 302 (1987), 77-86.

[BRR] L. Bukovsky, I. Reclaw, and M. Repicky, Spaces not distinguishing pointwise and quasinormal convergence of real functions, Topology Appl. 41 (1991), 25-40.

[CM] J. Cichon and M. Morayne, Universal functions and generalized classes of function, Proc. Amer. Math. Soc. 102 (1988), 83-89.

[CMPS] J. Cichon, M. Morayne, J. Pawlikowski, and S. Solecki, Decomposing Baire functions, J. Symbolic Logic 56 (1991), 1273-1283.

[L] N. Lusin, Sur les ensembles toujurs de premiere categorie, Fund. Math. 21 (1933), 114-126.

[M] A. W. Miller, Special subsets of the real line, Handbook of Set Theoretic Topology (K. Kunen and J. Vaughan, eds.), North-Holland, Amsterdam, 1984.

[Ma] E. Marczewski (Szpilrajn), Sur une classe de fonctions de W. Sierpiniski et la classe correspondante d'ensembles, Fund. Math. 24 (1935), 17-24.

[Sh] Juichi Shinoda, Some consequences of Martin's Axiom and the continuum hypothesis, Nagoya Math. J. 49 (1973), 117-125.

[S] W. Sierpiński, Sur un probleme concernant les fonctions semi-continues, Fund. Math. 28 (1937), 1-6.

[SZ] W. Sierpiński and A. Zygmund, Sur une fonction qui est discontinue tout ensemble de puissance de continu, Fund. Math. 4 (1923), 316-318.

[W] Elżbieta Wagner, Sequences of measurable functions, Fund. Math. 112 (1981), 89-102.

Institute of Mathematics, University of Gdańsk, Wita Stwosza 57, 80-952 Gdańsk, Poland

E-mail address: matir@halina.univ.gda.pl 